\title{
PANCREATITE AGUDA EXPERIMENTAL INDUZIDA PELA L-ARGININA: avaliação histológica e bioquímica
}

\author{
Odery RAMOS Jr. ${ }^{1}$, Olival Ronald LEITÃO ${ }^{1}$, \\ João Carlos Domingues REPKA ${ }^{1}$ e Sérgio Gabriel da Silva BARROS ${ }^{2}$
}

RESUMO - Racional - Doses excessivas de aminoácidos básicos como a L-arginina têm a capacidade de lesar o pâncreas de ratos. Objetivo - Descrever e avaliar as características bioquímicas e histológicas da pancreatite aguda induzida pela L-arginina em ratos durante a instalação, desenvolvimento e reparação do processo inflamatório pancreático. Material e Métodos - A amostra constituiu-se de 105 ratos machos, da linhagem Wistar. Aos ratos do grupo experimento $(\mathrm{n}=70)$ administrou-se injeção intraperitonial de $500 \mathrm{mg} / 100 \mathrm{~g}$ de peso corporal de L-arginina. No grupo controle $(\mathrm{n}=35)$ foi injetada solução salina isotônica. Analisaram-se 10 animais do grupo experimento e 5 do grupo controle em cada período de 6 h, 12 h, 24 h, 48 h, 72 h, 7 dias e 14 dias. Durante os tempos determinados coletou-se sangue para exames laboratoriais e o pâncreas para análise em microscopia óptica. Resultados - Doze a 24 horas após a injeção de L-arginina os níveis séricos de amilase atingiram valores máximos, comparados àqueles dos ratos controle, decrescendo gradualmente, alcançou-os na $48^{\mathrm{a}}$ hora sendo significativamente menor após 72 horas e 7 dias. A atividade enzimática retornou a níveis basais após 14 dias. Os valores de amilase estavam normais em todos os tempos avaliados nos animais do grupo controle. Na microscopia óptica, após injeção de L-arginina, observou-se arquitetura pancreática histologicamente preservada no período de 6 horas, evidenciando-se em 24 horas importante edema intersticial. Após 48 horas, a arquitetura acinar estava parcialmente destruída com necrose celular focal, atingindo sua máxima severidade ao ultrapassar 72 horas. No 7o dia a necrose tecidual e o edema haviam diminuído, iniciando-se a regeneração da arquitetura acinar. Observou-se a reconstrução estrutural pancreática após 14 dias. No grupo controle não se encontraram alterações histológicas pancreáticas. Conclusão - A pancreatite aguda experimental induzida pela L-arginina induz a necrose pancreática, apresentando evolução auto-limitada com regeneração do pâncreas em 2 semanas.

DESCRITORES - Pancreatite. Doença aguda. Arginina. Ratos.

\section{INTRODUÇÃO}

A pancreatite aguda pode apresentar-se como um distúrbio suave e autolimitado, ou sob a forma de doença grave de evolução muitas vezes fatal ${ }^{(21,25)}$. Apesar das modernas tecnologias disponíveis ${ }^{(4,8,17)}$, ainda persistem dúvidas acerca de inúmeros aspectos da patogenia e fisiopatologia da doença e, portanto, da melhor terapêutica a ser $\operatorname{adotada}^{(3,21)}$. Baseados nestes fatos, pesquisadores têm buscado métodos de indução de pancreatite através de meios experimentais em diferentes espécies animais ${ }^{(2,}$ 3, 13, 22). O modelo adequado deve ser tecnicamente simples e possuir características evolutivas que provoquem no animal alterações pancreáticas semelhantes àquelas observadas em seres humanos.

Com esse propósito, executou-se um modelo de pancreatite aguda experimental que utiliza a L-arginina, aminoácido essencial, barato e disponível no comércio, que administrado por via intra-peritonial, desencadeia pancreatite aguda em $\operatorname{ratos}^{(6,12,16,19,23,24)}$ e permite estudar as alterações histológicas e bioquímicas durante a instalação, desenvolvimento e reparação do processo inflamatório pancreático.

\section{MATERIAL E MÉTODOS}

Animais de experimentação

Caracterização da amostra - Utilizaram-se no experimento 105 ratos Wistar (Rattus norvegicus - Rodentia - Mammalia) machos, albinos não-isogênicos, com peso compreendido entre 182,4 g $\pm 12,6 \mathrm{~g}$, fornecidos pelo Biotério do Instituto de Tecnologia do Paraná, Curitiba, PR, e manipulados conforme normas para trabalhos experimentais publicadas no livro de GOLDIM e RAYMUNDO ${ }^{(10)}$.

\section{Ambiente de experimentação}

O experimento deste estudo foi executado no Laboratório de Microbiologia e Imunologia da Faculdade Evangélica de Medicina do Paraná, Curitiba, PR.

Apresentado como parte dos requerimentos para obter o Título de Mestre pela Faculdade de Medicina da Universidade Federal do Rio Grande do Sul - Área de Concentração em Gastroenterologia, Porto Alegre, RS.

${ }^{1}$ Faculdade Evangélica do Paraná, Curitiba, PR.; ${ }^{2}$ Faculdade de Medicina da Universidade Federal do Rio Grande do Sul, Porto Alegre, RS.

Endereço para correspondência: Dr. Odery Ramos Júnior - Rua Mal. Deodoro, 497 - 80020-909 - Curitiba - PR. E-mail: odery@netpar.com.br 


\section{Delineamento experimental}

Dividiu-se a amostra em 2 grupos, conforme demonstrativo abaixo:

\begin{tabular}{|c|c|c|}
\hline Grupo & Objetivo & Aferições \\
\hline I & $\begin{array}{l}\text { 70 ratos que receberam por via intraperitonial, } \\
\text { solução de L-arginina na dose de } 500 \mathrm{mg} / 100 \mathrm{~g} \\
\text { de peso corpóreo. Foram sacrificados em grupos de } \\
10 \text { animais, respectivamente, às } 6 \mathrm{~h}, 12 \mathrm{~h}, 24 \mathrm{~h}, \\
48 \mathrm{~h}, 72 \mathrm{~h}, 7 \text { dias e } 14 \text { dias, a partir da inoculação. } \\
\text { Testes animais, eram coletadas amostras de sangue } \\
\text { para determinações bioquímicas e fragmentos de } \\
\text { pâncreas para análise histológica }\end{array}$ & Dosagem de amilase \\
\hline II & $\begin{array}{l}35 \text { ratos que receberam por via intraperitonial, o } \\
\text { volume de solução fisiológica equivalente ao volume } \\
\text { de solução de L-arginina, aleatoriamente, divididos pâncreas para } \\
\text { em grupos de } 5 \text { animais, que eram sacrificados nos } \\
\text { mesmos tempos do grupo teste, para dosagens } \\
\text { bioquímicas e histologia de controle }\end{array}$ & Dosagens de amilase \\
Controle & Retirada de pâncreas \\
para histologia
\end{tabular}

\section{Injeção da L-arginina}

Preparo da soluçáo - Foram pesados $20 \mathrm{~g}$ de L-arginina (Merck® artigo 1.01542), diluindo-se em volume final de $100 \mathrm{~mL}$ de solução fisiológica, ajustando-se o pH entre 6,8-7,4 com solução tampão de fosfatos. Esterilização em membranas de $0,22 \mu$ (Millipore ${ }^{\circledR}$ ). Preparo imediato antes do uso.

Injeção por via intraperitonial - Ratos machos Wistar nutridos com alimentação específica para a espécie ad libitum, eram distribuídos aleatoriamente em caixas de polipropileno identificadas, com cinco animais cada e que foram deixados em jejum durante a noite prévia ao experimento. Após pesagem, os animais receberam injeção intraperitonial de $500 \mathrm{mg} / 100 \mathrm{~g}$ de peso corporal de L-arginina. $\mathrm{O}$ volume total calculado foi dividido em doses iguais e injetado em duas etapas com intervalos de 15 minutos, em um dos quadrantes inferiores abdominais. Os ratos controle receberam o mesmo volume de solução fisiológica $0.15 \mathrm{M}$. Todos os animais receberam alimentação à vontade após a injeção.

\section{Coleta de amostras e sacrifício de animais}

Análise laboratorial - Para a coleta de sangue, os animais foram anestesiados por inalação com éter sulfúrico e cuba plástica apropriada. A seguir, foram colocados em decúbito dorsal e com seringa de $10 \mathrm{~mL}$ e agulha de $25 \times 8 \mathrm{~mm}$ procedia-se à punção cardíaca transtorácica. O sangue coletado foi centrifugado a 700 rpm durante 10 minutos, separando-se a seguir o soro para as determinações de amilase através do método enzimático cinético a $405 \mathrm{~nm}$, efetuadas em equipamento automatizado - Sistema COBAS - MIRA "S".

Análise Histológica - Após a coleta de sangue, os animais foram recolocados em cuba sob inalação letal de éter sulfúrico. Posteriormente ao sacrifício, os animais foram submetidos a abertura ampla da cavidade abdominal, sendo retirado o pâncreas para avaliação histológica.

O pâncreas retirado era colocado em formaldeído a $10 \%$ pelo período mínimo de 48 horas para adequada fixação. Secções foram feitas através do longo eixo da glândula, coradas com hematoxilina-eosina e examinados por microscopia óptica ${ }^{(2)}$. Todos os cortes histológicos foram examinados sem o conhecimento do grupo a que pertenciam e ao período em análise. Foram considerados os seguintes padrões histológicos: edema, infiltração inflamatória, hemorragia e necrose parenquimatosa ${ }^{(20,24)}$. O padrão histológico foi definido de acordo com a presença e predominância das alterações microscópicas descritas no Quadro 1.

QUADRO 1 - Critérios histológicos para a avaliação da pancreatite

\begin{tabular}{|l|c|c|c|c|}
\hline Edema & ausente & leve & moderada & Severa \\
\hline $\begin{array}{l}\text { Infiltração } \\
\text { inflamatória }\end{array}$ & ausente & leve & moderada & Severa \\
\hline $\begin{array}{l}\text { Necrose } \\
\text { parenquimatosa }\end{array}$ & ausente & $\begin{array}{c}\text { leve } \\
\text { (focal) }\end{array}$ & $\begin{array}{c}\text { moderada } \\
\text { (difusa) }\end{array}$ & $\begin{array}{c}\text { Severa (extra- } \\
\text { parenquimatosa; adiposa) }\end{array}$ \\
\hline Hemorragia & ausente & leve & moderada & Severa \\
\hline
\end{tabular}

\section{Estudo estatístico}

Nas comparações entre dois grupos independentes, o teste aplicado foi o de Mann-Whitney e quando da comparação de mais de dois grupos independentes, o teste aplicado foi o de Kruskal-Wallis. Em todos os testes foi adotado o nível de significância de $P \leq 5 \%{ }^{(7,9)}$.

\section{RESULTADOS}

\section{Níveis de Amilase}

Na Tabela 1 são apresentados, para cada grupo e em cada momento analisado, as médias, os desvios-padrão e os valores de $P$ obtidos através da aplicação do teste de Mann-Whitney.

TABELA 1 - Níveis de amilase: médias, desvios padrão e valores de $P$ nas comparações de grupos

\begin{tabular}{lccc}
\hline \multirow{2}{*}{ PERÍODOS } & \multicolumn{2}{c}{ GRUPO } & \multirow{2}{*}{ Valor de $\boldsymbol{P}$} \\
\cline { 2 - 3 } & Média $\pm \mathrm{DP} *$ & Média $\pm \mathrm{DP}$ & \\
\cline { 2 - 3 } 6 horas & $984.2 \pm 77.6$ & $5238.2 \pm 1863.6$ & 0.0022 \\
12 horas & $932.2 \pm 164.7$ & $8647.1 \pm 3313.8$ & 0.0022 \\
24 horas & $977.6 \pm 88.1$ & $3327.2 \pm 1032.7$ & 0.0022 \\
48 horas & $1022.4 \pm 89.9$ & $1025.9 \pm 171.1$ & 0.9025 \\
72 horas & $1042.4 \pm 114.8$ & $707.6 \pm 151.0$ & 0.0048 \\
7 dias & $1074.0 \pm 152.5$ & $796.3 \pm 93.2$ & 0.0084 \\
14 dias & $1054.2 \pm 84.8$ & $1011.7 \pm 66.8$ & 0.1779 \\
\hline
\end{tabular}

*DP $=$ Desvio padrão

Observou-se que na avaliação realizada nos períodos de 6 , 12, 24, 72 horas e 7 dias, as médias dos valores de amilase nos grupos controle e experimento são significativamente diferentes ao nível de $5 \%$.

O Gráfico 1 apresenta a evolução dos valores médios de amilase ao longo dos períodos analisados nos dois grupos. 
GRÁFICO 1 - Médias dos níveis de amilase nos grupos controle e experimento em cada tempo do estudo

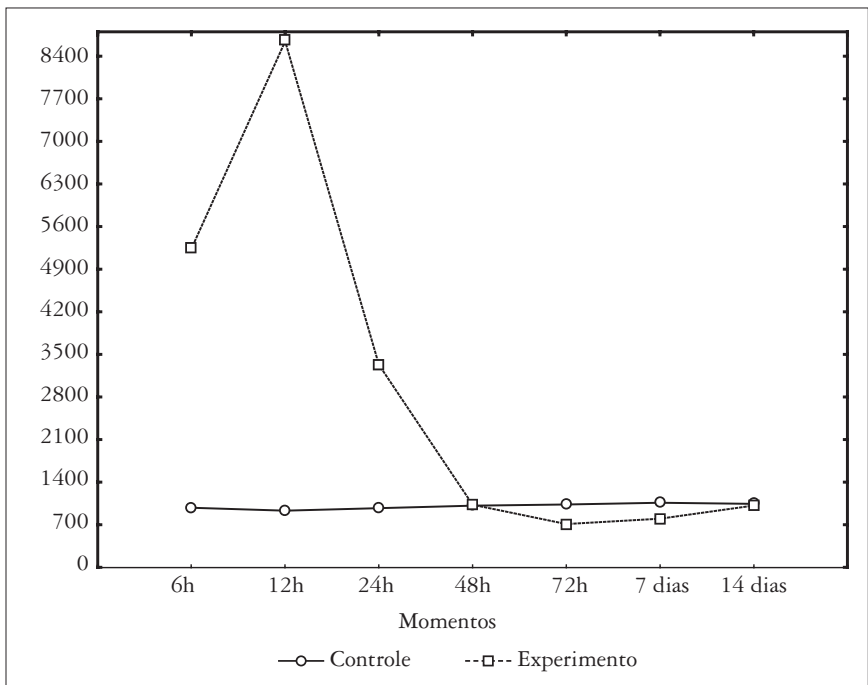

\section{Exame histológico}

Para a análise dos exames histológicos no grupo experimento, os resultados foram ordenados de acordo com as diversas combinações dos níveis de gravidade de edema e necrose, da situação menos grave para a mais grave (de 1 a 13), determinando-se estas ordenações por graus. Os números de casos observados, segundo esta ordenação, e os graus médios em cada período do estudo são apresentados na Tabela 2.

O Gráfico 2 apresenta os graus médios (gravidade) relativos ao exame histológico para cada um dos momentos do estudo.

Analisando os resultados dos testes e os graus médios, observou-se que a situação histológica agravou-se significativamente a partir de 6 horas até 24 horas. De 24 horas a 48 horas, não se observou diferença

TABELA 2 - Freqüência de casos observados em ordem segundo a gravidade no exame histológico no grupo experimento

\begin{tabular}{|c|c|c|c|c|c|c|c|c|}
\hline \multicolumn{2}{|c|}{$\begin{array}{c}\text { Ordenação segundo a } \\
\text { gravidade (graus) }\end{array}$} & \multirow{2}{*}{$\frac{6 \mathrm{~h}}{10}$} & \multirow[t]{2}{*}{$12 \mathrm{~h}$} & \multirow[t]{2}{*}{$24 \mathrm{~h}$} & \multirow[t]{2}{*}{$48 \mathrm{~h}$} & \multirow[t]{2}{*}{$72 \mathrm{~h}$} & \multirow[t]{2}{*}{7 dias } & \multirow{2}{*}{$\begin{array}{c}14 \\
\text { dias } \\
9\end{array}$} \\
\hline & 1 & & & & & & & \\
\hline 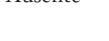 & 2 & & 8 & 1 & & & 3 & 1 \\
\hline & 3 & & 2 & 3 & & & & \\
\hline \multirow[t]{4}{*}{ Leve } & 4 & & & & & & & \\
\hline & 5 & & & & 1 & & & \\
\hline & 6 & & & & 3 & & & \\
\hline & 7 & & & & & 1 & & \\
\hline \multirow[t]{4}{*}{ Moderado } & 8 & & & 3 & 1 & & & \\
\hline & 9 & & & & 4 & 3 & 6 & \\
\hline & 10 & & & & 1 & 2 & 1 & \\
\hline & 11 & & & 3 & & & & \\
\hline \multirow[t]{2}{*}{ Grave } & 12 & & & & & & & \\
\hline & 13 & & & & & 4 & & \\
\hline \multicolumn{2}{|c|}{ GRAUS MÉDIOS } & 1 & 2.2 & 6.8 & 7.7 & 10.6 & 7.0 & 1.1 \\
\hline
\end{tabular}

GRÁFICO 2 - Graus médios relativos à gravidade do exame histológico no grupo experimento

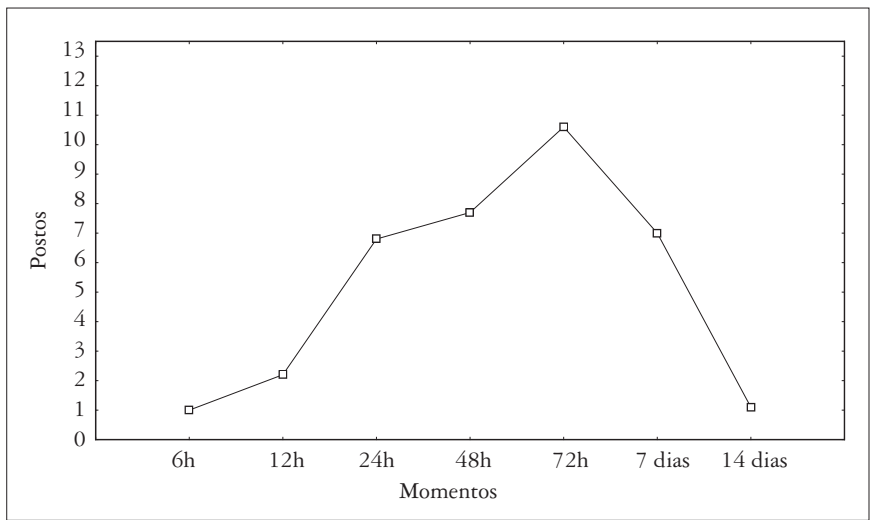

significativa nos aspectos histológicos. A histologia demonstrou aspecto de agravamento no período compreendido entre 48 e 72 horas e melhora progressiva do $3^{\circ}$ dia para 7 dias e 14 dias.

No grupo controle, a análise microscópica do parênquima pancreático estava histologicamente preservada em todos os momentos considerados. As Figuras 1 e 2 mostram fotos do pâncreas após injeção de L-arginina.

\section{DISCUSSÃO}

TANI et al. ${ }^{(24)}$, demonstraram novo modelo experimental de pancreatite aguda necrotizante em ratos induzido por uma única injeção intraperitonial 9+de L-arginina. Em trabalhos anteriores, neste laboratório, observou-se que a injeção de L-arginina em dose única de $500 \mathrm{mg} / 100 \mathrm{~g}$ de peso corpóreo, em ratos pesando entre $350 \mathrm{~g}$ e $450 \mathrm{~g}$, produz mortalidade em decorrência de trombose mesentérica. Assim, no presente estudo, a dose total foi dividida em duas injeções, com intervalo de 15 minutos e os ratos selecionados pesando, desta vez, $130 \mathrm{~g}$ a $150 \mathrm{~g}$, com o intuito de excluir tal intercorrência.

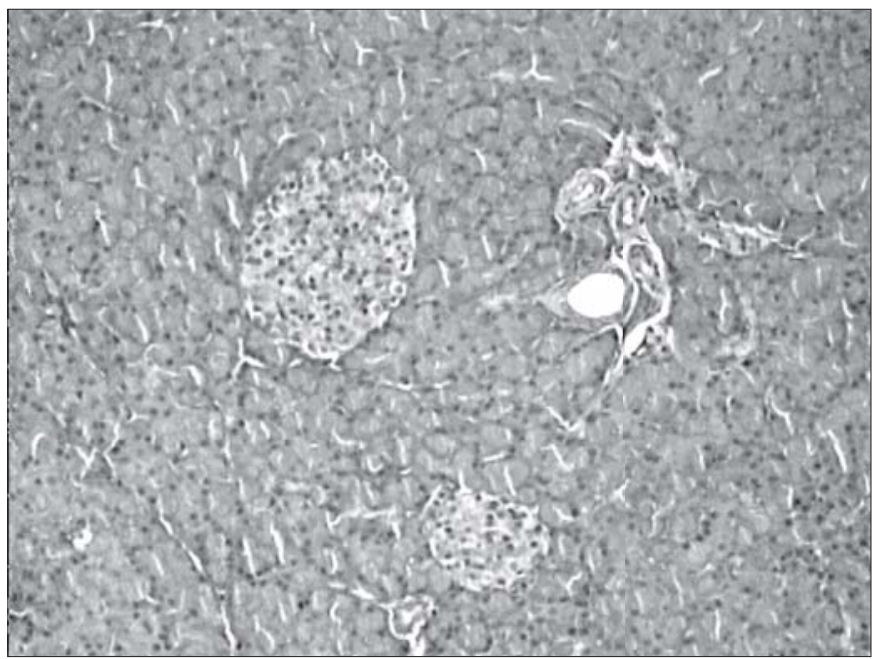

FIGURA 1 - Pâncreas de um animal 6 horas após injeção de L-arginina. A arquitetura glandular está histologicamente preservada $(\mathrm{H}-\mathrm{E} \times 100)$ 


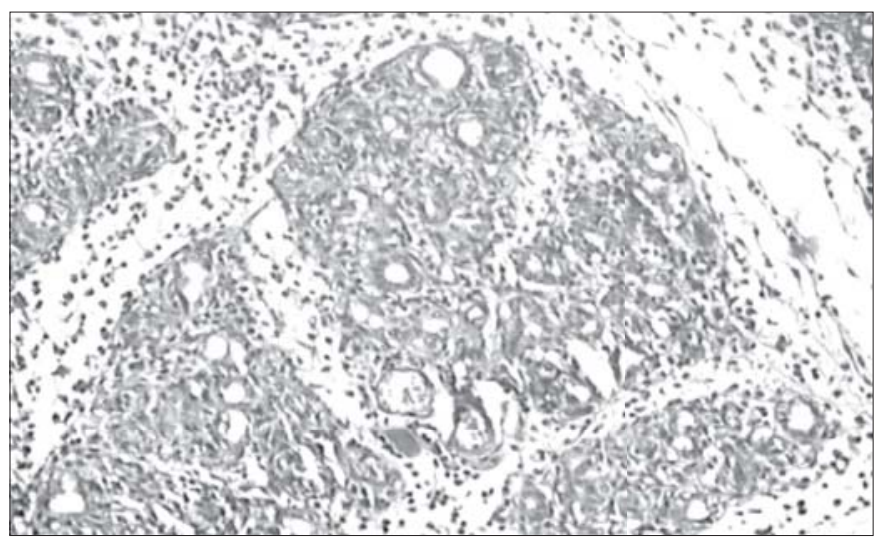

FIGURA 2 - Quadro histológico 72 horas após injeção de L-arginina, marcante alteração no parênquima pancreático. Extensa degeneração ou necrose de células acinares e infiltrado inflamatório intersticial $(\mathrm{H}-\mathrm{E} \times 100)$

Neste estudo, os níveis de amilase sérica permaneceram constantes nos animais do grupo controle, mas elevaram-se significativamente nos animais que receberam injeção de L-arginina. Todos os animais que receberam L-arginina intraperitonial demonstraram aumento na atividade da amilase sérica mais pronunciado no período de 12 horas e retornaram aos níveis dos controles em 48 horas. Embora os níveis séricos de amilase tenham demonstrado maior elevação entre 12 e 24 horas após a injeção de L-arginina, a maioria das alterações severas, como a necrose de células acinares, foi encontrada em 72 horas. A significativa redução de síntese das enzimas pancreáticas, em período subseqüente às 72 horas após indução de pancreatite, pode resultar em diminuição dos seus níveis séricos. Nesta etapa, observou-se que os valores de amilase foram significativamente menores nos ratos do grupo com pancreatite, quando comparados com o controle no período de 72 horas e que retornaram aos níveis basais em 2 semanas, acompanhando a regeneração pancreática, como evidenciado pelos padrões histológicos neste período de evolução.

Estes achados permitem referir que os níveis de amilasemia não se correlacionam diretamente com a gravidade da inflamação. A maior parte dos autores são concordes quanto à especificidade da elevação da amilase sérica ser deficiente como valor de prognóstico da pancreatite aguda $^{(18)}$. Até mesmo a correlação inversa entre este parâmetro e a severidade da lesão pancreática aguda, como observada nos períodos de 72 horas e 7 dias deste experimento, foram descritas e atribuídas ao declínio na capacidade de secreção quando o dano celular pancreático é severo ${ }^{(1,5)}$. Esta análise é compatível com a observação de que os níveis mais altos de amilase sérica são encontrados nos modelos experimentais de pancreatite caracterizados por edema intersticial e morte mínima de células ${ }^{(11)}$. No presente estudo e em concordância com estudo previamente realizado ${ }^{(20)}$, o edema pancreático, que não é indicador de severidade, demonstrou melhor correlação com a hiperamilasemia, sendo que os índices usuais para o diagnóstico de pancreatite severa, como necrose acinar, apresentam correlação inadequada ou mesmo inversa com o nível da amilase sérica.
No exame histológico, após a injeção de L-arginina durante os períodos de 6, 12 e 24 horas, verificou-se infiltração progressiva no interstício e tecido pancreático de leucócitos polimorfonucleares, linfócitos e macrófagos. Após 6 horas da aplicação de L-arginina, não se observaram edema intersticial pancreático ou necrose de células acinares. Após 12 horas, observou-se edema intersticial com discreta infiltração de células inflamatórias e, decorridas 24 horas, evidenciaram-se ácinos separados por importante edema intersticial. No período entre 24 e 48 horas não ocorreram alterações histológicas significativas.

Evidenciou-se que, a partir do $2^{\circ}$ dia, iniciaram-se focos de necrose nas células acinares e que a necrose comprometia grande parte do parênquima, provocando desordem da arquitetura pancreática, lesões de células acinares e intenso processo inflamatório agudo ao progredir de 72 horas. Nesta fase do estudo, constatou-se que as células acinares pancreáticas haviam sido progressivamente destruídas, que os ductos pancreáticos encontravam-se dilatados e repletos de muco, enquanto as ilhotas de Langerhans pareciam preservadas. O estudo histológico, 7 dias após a aplicação de Larginina, mostrou melhora considerável do edema e da necrose pancreática. Embora se devam considerar as dificuldades em estimar a percentagem de extensão da necrose tecidual, os baixos níveis de enzimas pancreáticas séricas nestes períodos sugerem significativa necrose das células acinares. Ao final do estudo observou-se, pela histologia do $14^{\circ}$ dia pós-injeção de L-arginina, que a arquitetura original pancreática havia sido restaurada.

Trabalhos recentes utilizando modelo experimental de pancreatite aguda induzida pela L-arginina em ratos, sugerem que esta induz apoptose e expressão do gene da proteína associada à pancreatite em célula acinar pancreática ${ }^{(14)}$. Outras alterações, como ativação NFKappa $\mathrm{B}^{(19)}$, fator de crescimento fibroblástico FGF-7 e FGF-10 ${ }^{(12)}$, efeito exercido pela L-arginina como precursora do óxido nítrico no sistema circulatório e, mesmo, pela regulação da circulação pancreática $^{(15,23)}$, bem como o papel de radicais livres derivados do oxigênio têm sido estudadas na patogênese da inflamação e da fibrose pancreática ${ }^{(6,16)}$.

Enfatiza-se que, embora a patogênese da pancreatite aguda induzida pela L-arginina permaneça indefinida, a metodologia empregada neste modelo experimental mostra-se útil e torna-se alternativa para o estudo da mesma.

\section{CONCLUSÕES}

Provocando-se pancreatite aguda em ratos machos da raça Wistar, pela injeção intraperitonial de L-arginina através da metodologia empregada e baseado nos resultados obtidos, concluiu-se que:

Os níveis séricos de amilase mostraram-se capazes de contribuir para o diagnóstico da pancreatite aguda, porém não se correlacionam com a gravidade das lesões pancreáticas.

A administração de L-arginina na dose $500 \mathrm{mg} / 100 \mathrm{~g}$ de peso corpóreo induz pancreatite aguda necrotizante que por não ser fatal, permite avaliar todas as fases da inflamação pancreática aguda, ou seja, desde sua instalação à regeneração do órgão. 
Ramos Jr O, Leitão OR, Repka JCD, Barros SGS. Experimental acute pancreatitis induced by L-arginine - a histological and biochemical evaluation. Arq Gastroenterol 2005;42(1):55-9.

ABSTRACT - Background - Excessive doses of basic amino acids such as L-arginine are able to injure the pancreas of rats. Aim - To describe and evaluate the biochemical and histological characteristics of acute pancreatitis in rats induced by L-arginine during the installation, development and repair stages of the pancreatic inflammatory process. Material and Methods - The study group consisted of 105 male Wistar rats. The rats in the experimental group (n $=70)$ received $500 \mathrm{mg} / 100 \mathrm{~g}$ of corporal weight L-arginine injection intraperitoneally. In the control group $(\mathrm{n}=35)$, isotonic saline solution was injected. Ten rats in the experiment group and five in the control group were analyzed after $6,12,24,48$, and 72 hours, on the $7^{\text {th }}$ and $14^{\text {th }}$ days. During those times, blood samples were collected for laboratory testing and samples from the pancreas were collected for an optical microscopy analysis. Results - From 12 to 24 hours after the injection of L-arginine, the amylase serum levels raised to their peak values when compared to the rats in the control group, decreasing gradually, reaching an equal level after the $48^{\text {th }}$ hour and being significantly lower after 72 hours and 7 days. The enzymatic activity returned to its basal level after 14 days. The amylase values were normal in all the times evaluated in the control group. In optical microscopy, after the injection of L-arginine, a pancreatic architecture histologically preserved was observed after 6 hours, evidencing an important interstitial edema in 24 hours. After 48 hours, the acinar architecture was partially destroyed with focal cellular necrosis, reaching its maximum severity after 72 hours. On the $7^{\text {th }}$, the tissue necrosis and the edema had diminished, and the regeneration of the acinar architecture initiated. The pancreatic structural reconstruction could be observed after 14 days. Pancreatic histological changes were not found in the control group. Conclusion - Experimental acute pancreatitis induced by L-arginine leads to pancreatic necrosis showing self-limited evolution with pancreas regeneration in 2 weeks.

HEADINGS - Pancreatitis. Acute disease. Arginine. Rats.

\section{REFERÊNCIAS BIBLIOGRÁFICAS}

1. Adams JT, Libertino JA, Schwartz SI. Significance of an elevated serum amylase. Surgery 1968;63:877-84.

2. Aho HJ, Nevalainem TJ. Experimental pancreatitis in the rat. Light and electron microscopical studies on early pancreatic lesions induced by intraductal injection of trypsin, phospholipase $\mathrm{A}^{2}$, lysolecythin and non-ionic detergent. Virchows Arch B Cell Pathol 1982;40:347-56.

3. Banerjee AK, Galloway SW, Kingsnorth NA. Experimental models of acute pancreatitis. Br J Surg 1994;81:1096-1103.

4. Clavien PA, Hauser H, Meyer P, Rohner A. Value of contrast computerized tomography in the early diagnosis and prognosis of acute pancreatitis. Am J Surg 1988;155:457-66.

5. Clavien PA, Robert J, Meyer P, Borst F, Hauser H, Herrmann F, Dunand V, Rohner A. Acute pancreatitis and normoamylasemia. Not an uncommon combination. Ann Surg 1989;210:614-20.

6. Czakó L, Takács T, Varga IS, Tiszlavicz L, Hai DQ, Hegyi P, Matkovics B, Lonovics J. Involvement of oxygen-derived free radicals in L-arginine-induced cute pancreatites. Dig Dis Sci 1998;43:1770-7.

7. Everitt BS. $2 \times 2$ contingency tables. In: Everitt BS, editor. The analysis of contingency tables. London: Chapman and Hall; 1977. p.12-37.

8. Fan ST, Lai EC, Mok FP, Lo CM, Zheng SS, Wong J. Early treatment of acute biliary pancreatitis by endoscopic papillotomy. N Engl J Med 1993;328:228-32.

9. Fletcher RH, Fletcher SW, Wagner EH. Diagnóstico. In: Fletcher RH, Fletcher SW, Wagner EH, editor. Epidemiologia clínica: elementos essenciais. 3.ed. Porto Alegre: Artes Médicas; 1996. p.52-83.

10. Goldim JR, Raymundo MM. Pesquisa em saúde e direito dos animais. Porto Alegre: HCPA; 1997.

11. Gullick HD. Relation of the magnitude of blood enzyme elevation to severity of exocrine pancreatic disease. Am J Dig Dis 1973;18:375-83.

12. Ishiwata T, Naito Z, Lu YP, Kawahara K, Fujii T, Kawamoto Y, Teduka K, Sugisaki Y. Differential distribution of fibroblast growth factor (FGF)-7 and FGF-10 in Larginine-induced acute pancreatites. Exp Mol Pathol 2002;73:181-90.

13. Lerch MM, Adler G. Experimental animal models of acute pancreatitis. Int J Pancreatol 1994;15:159-70.

14. Motoo Y, Taga K, Su SB, Xie MJ, Sawabu N. Arginine induces apoptosis and gene expression of pancreatites-associated protein (PAP) in rat pancreatit acinar AR4-2J cells. Pancreas 2000;20:61-6.
15. Nakaki T, Kato R. Beneficial circulatory effect of L-arginine. Jpn J Pharmacol 1994;66:167-71.

16. Naito Z, Ishiwata T, Lu YP, Teduka K, Fujii T, Kawahara K, Sugisaki Y. Transient and ectopic expression of lumican by acinar cells in L-arginine-induced acute pancreatites. Exp Mol Pathol, 2003;74:33-9.

17. Neoptolemos JP, Carr-Locke D1, London NJ, Bailey IA, James D. Controlled trial of urgent endoscopic retrograde cholangiopancreatography and endoscopic sphincterotomy versus conservative treatment for acute pancreatitis due to gallstones. Lancet 1988;2:979-83.

18. Puolakkainen PA. Early, assessment of acute pancreatitis. A comparative study of computed tomography and laboratory tests. Acta Chir Scand 1988;155:25-30.

19. Rakonczay Z, Jármay K, Kaszaki J, Mándi Y, Duda E, Hegyi P, Boros I, Lonovics J, Takács T. NF-kappaB activation in arginine-induced acute pancreatitis. Free Radic Biol Med 2003;34:696-709.

20. Schmidit J, Lewandrowski C, Del Castilho F, Mandavilli U, Compton CC, Warshaw $\mathrm{AL}$, rattner DW. Histopathologic correlates of serum amylase activity in acute experimental pancreatitis. Dig Dis Sci 1992;37:1426-33

21. Steer ML. Classification and pathogenesis of pancreatitis. Surg Clin North Am 1989;101:1701-9.

22. Sum PT, Bencosme AS, Beck IT. Pathogenesis of bile-induced acute pancreatitis in the dog: experiments with detergents. Am J Dig Dis 1970;15:637-46.

23. Takácz T, Czakó L, Morschl E, Lászlo F, Tiszlavicz L, Rakonczay Z, Lonovicz J. The role of nitric oxide in edema formation in $\mathrm{L}$-arginine-induced acute pancreatitis. Pancreas 2002;25:277-82

24. Tani S, Itoh H, Okabayashi Y, Nakamura T, Fujii M, Fujusawa T, Koide M, Otsuki M. New model of acute necrotizing pancreatitis induced by excessive doses of arginine in rats. Dig Dis Sci 1990;35:367-74.

25. Varga IS, Matkovics B, Czako L, Hai DQ, Kotorman M, Takacz T, Sasvari M. Oxidative stress changes in L-arginine-induced pancreatitis in rats. Pancreas 1997;14:355-9. 